\title{
"Ich werde ja keine Morpheme übersetzen". Sprache und sprachwissenschaftliche Lehre als Problem und Herausforderung in der Übersetzungsausbildung
}

\author{
Carsten Sinner, sinner@uni-leipzig.de \\ Elia Hernández Socas, socas@uni-leipzig.de \\ Héctor Hernández Arocha, hharocha@googlemail.com \\ Universität Leipzig \\ https://dx.doi.org/10.12795/futhark.2014.i9.17
}

Zusammenfassung: Der Beitrag setzt sich kritisch mit den Konsequenzen der kulturellen Wende, den Postulaten der "postlinguistischen" Übersetzungswissenschaft auseinander und diskutiert die Rolle, die sprachwissenschaftliche Ansätze in Ausbildung und Forschung einnehmen können bzw. in einigen Bereichen der Übersetzungsausbildung allgemein und in der Ausbildung im Sprachenpaar Deutsch und Spanisch speziell einnehmen müssten.

Stichwörter: Sprachwissenschaft, Übersetzungsausbildung, Sprachenpaar Deutsch-Spanisch.

Abstract: The article critically examines the implications of the cultural turn as well as the postulates of "post-linguistic" translation studies, and discusses the role of linguistic approaches in translator training and research, and which role they should have in specific areas of translator training in general and for the language pair German-Spanish in particular.

Key words: Linguistics, translator training, language pair GermanSpanish. 
Die Auseinandersetzung mit den der Arbeitssprachaft und der einzelsprachliagen en, aber translationsrelevanten sper diese Grund $\mathrm{Sprach}_{w_{1}} \mathrm{Al}_{\mathrm{g}} \mathrm{m}_{\mathrm{mein}}$ seit den Anfängen der universitären Ausbildung velichen Ausgeh aff schlägige linguistische Komponenten in dem Maße in dirden Hochschulen anstrebten die Einrichtungen eine An die Leh $\mathrm{ei}_{\mathrm{i}}$

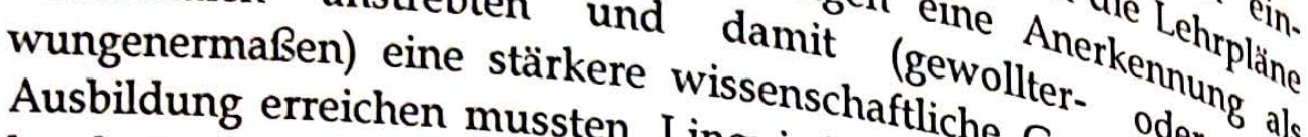
Ausbildung erreichen mussten. Linguischaftliche $\mathrm{Grund}_{\text {oder }} \mathrm{g}$ als hende Kenntnisse der Arbeitssprachistische Kenntnisslegung gez.

Modellen der Translationskompetenz aufgeführt.
Tatsächlich wird die sprachwissensch ung tief $\mathrm{der}_{\mathrm{g}}$
allen

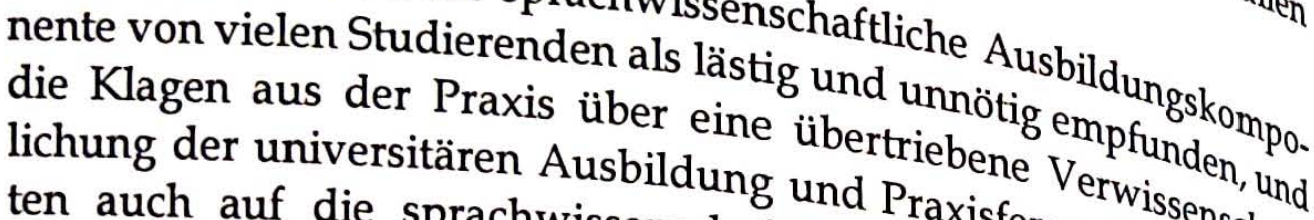
lichung der universitären Ausbildung übertriebene Verwissenschaft. ten auch auf die sprachwissenschaftlichen Ausbildungelt nicht sel.
Insbesondere haben nicht zuletzt auch die kulturelle Wenteile ab.
Translationswissenschaft und die Darstellung Translationswissenschaft und die Darstellung der seit dende in der Jahren verstärkt propagierten translatorischen Ansätzen den $1980 \mathrm{er}$ Postulat, die "Überwindung" der vermeintlichen Lime mir ihrem linguistischen Richtungen in der Translationswish Limitationen der haben, nach den WissenschaftlerInnen auch den Sthaft erreicht ${ }_{24}$ mente gegen eine vertiefende linguistische Aen Studierenden Arguduktion des sprachwissenschaftlichen Anteils nalyse und für eine Re. bildung gegeben.

Wir möchten uns kritisch mit den Konsequenzen der kulturellen Wende, den Postulaten der "postlinguistischen" Übersetzungswissenschaft und den veränderten Voraussetzungen der StudienanfängerInnen für die Lehre in Übersetzung und Übersetzungswissenschaft und im weiteren Sinne für die translatorische Forschung auseinanderset. zen. Dabei geht es im engeren Sinne um die Rolle, die sprachwissenschaftliche Ansätze in Ausbildung und Forschung einnehmen können bzw. in einigen Bereichen der Übersetzungsausbildung allgemein und 
der Ausbildung im Sprachenpaar Deutsch und Spanisch speziell einnehmen müssten.

Als die übersetzungswissenschaftlichen Bemühungen in Leipzig, im Umfeld des später als Leipziger Schule bezeichneten Kollegiums begannen, interessierte man sich für die Regelmäßigkeiten und die Universalia beim Übersetzen. Ähnlich hat es die Stylistique comparée betrachtet, oder Autoren wie Koller, die tatsächlich den Übersetzern auf die Finger, bzw. in die Übersetzungen schauten, um zu Regeln, zu Regelmäßigkeiten zu kommen, die eben nicht an der Tagesform des Übersetzers, mit seinem schweren Abendessen, dem Wetter, seinen schlechten Sprachkenntnissen oder skurrilen Übersetzungsaufträgen zusammenhängen. Was passiert beim Übersetzen genau?, das war die Frage, die sie sich gestellt haben. Welche Erscheinungen werden immer wieder auftreten, werden immer wieder übersetzt, und wie haben Übersetzer dafür eine Lösung gefunden?

Auch diesen Autoren war schon klar, dass es Aspekte gibt, bei denen etwas ausgetauscht werden muss, weil es für den Übersetzungsauftrag nicht gebraucht wird, wo man etwas weglässt, etwas hinzufügt, Namen verändert und kulturelle Referenzen erläutert und dergleichen mehr. Aber sie waren sich ganz offensichtlich bereits über eines bewusst: Man kann diese individuellen Aspekte der Kultureingebundenheit und Dinge, die sozusagen nicht zu einem idealen Übersetzer passen, zwar systematisieren, aber nicht zusammenfassen zu Regeln und Regelmäßigkeiten, die man in der Lehre vermitteln kann und die hinausgehen würden über die reine Erwähnung der Klassen von Übersetzungsproblemen, die mit Kultur und mit den Umständen des Übersetzungsauftrags zu tun haben. Man kann sie auflisten, und das haben diese Autoren getan, beispielsweise die Kollegen aus Leipzig, als sie erklärt haben, womit sich die Linguistik nicht auseinandersetzen kann.

Snell-Hornby geht in ihrer Neuorientierung in den 1980er Jahren davon aus, dass die linguistische Perspektive in eine Sackgasse geraten war, was uns sehr extrem formuliert erscheint. Die Autoren der skopostheoretischen Ausrichtung waren und sind der Meinung, dass sie die linguistischen Ansätze übervunden haben, sehen sie also als etwas, das ein Hindernis darstellte. Nord lässt keine Gelegenheit aus zu ber-

Futhark 9 (2014) Sinner,Hernández,E., Hernández,H., Ich wende ja keine, 453-466 ISSN $1886-9300$ 
ichten, dass man mit Sprachwissenschaft beim Übersetzen nicht weiterkommt, so etwa in Gesprächen über Forschung in der Übersetzung, die 2011 in Leipzig im Rahmen der Leipziger Sommerschule zur Translation geführt wurden. Stolze verlangt, man soll sich identifizieren mit den Texten, die man übersetzt, und zwar mit ganzen Texten, nicht mit kurzen Übersetzungseinheiten wie etwa Sätzen oder Absätzen (und sie schießt nicht nur indirekt gegen die Linguisten, die sich ja auch "mit Sätzen" auseinandersetzen und abgesehen von der Textlinguistik - nicht explizit immer mit "ganzen Texten").

Auch Schreiber (2006: 34) zählt unterschiedliche Positionen zur Frage der Übersetzung und der Sprachwissenschaft auf, die den linguistischen Zugang zurückweisen, darunter vor allem die Positionen aus der Blickrichtung der literarischen Übersetzung. Unter den 10 Geboten für werdende Übersetzer von Truffaut (1983) findet sich etwa an erster Stelle auch eines, in dem die Linguistik ganz eindeutig aus der Übersetzungswissenschaft herausgehalten wird: „Linguistique et traduction tu distingueras".

Aber man sieht, wenn man ehrlich und unvoreingenommen an das Thema herangeht, dass linguistische Herangehensweisen auch bei der Übersetzung von Literatur nicht ganz unbeteiligt sind. Wenn ein deutscher, mit dem karibischen Spanisch vertrauter Übersetzer in einem Roman eines spanischen Autors auf Deutsch eine Gruppe junger Madrilenen, die in Madrid in einer Kneipe sitzen, gemeinsam Zuckerrohr knabbern lässt, weil er tomar cañas nicht anders verstehen kann, dann ist das nicht lediglich ein Beispiel für Kulturgebundenheit, sondern dann geht es außer um fehlendes Gespür dafür, was merkwürdig oder absurd ist, wohl auch um fehlende Recherchekompetenz; aber im Grunde geht es nur um fehlendes Vokabelwissen, um nicht ausreichende Kenntnisse der in diesem Fall einer bestimmten Varietät zugehörigen Lexik.

Wenn Literaten und Zeitungsautoren in Rezensionen etwa von deutschen Übersetzungen von Joseph Conrad darüber diskutieren, ob es besonders kreativ ist, wenn im Deutschen jemand um den Busch herum schlägt, weil im Original von Conrad beat about the bush zu lesen ist und man dadurch dann beim Lesen verstehe, wie Conrad schreibt,

Futhark 9 (2014) Sinner,Hernández,E., Hernández,H., Ich werde ja keine, 453-466 ISSN 1886-9300 
oder ob man eine Redewendung nicht besser durch eine deutsche Redewendung „ersetzen" solle, so ist für Linguisten - oder vielmehr für Übersetzungswissenschaftler, die nicht davor zurückschrecken, auch linguistische Blickwinkel zu berücksichtigen - klar, dass man eine Redewendung natürlich Wort für Wort übersetzen kann, wenn man denn Gründe dafür hat, dass aber eine Redewendung, nicht anders als ein Lexem, entweder bekannt ist oder nicht bekannt ist, und dass Wort für Wort übersetzte Redewendungen in erster Linie nun einmal an den Englischkenntnissen eines Übersetzers zweifeln lassen und nicht unbedingt Hinweis auf seine besondere Kreativität sind.

Schreiber ist der Meinung, dass "gerade die kontrastive Linguistik (d.h. der Sprachvergleich) hilfreich für das Übersetzen und Dolmetschen sein [kann], vor allem wenn sie zu einer lösungsorientierten, sprachbezogenen Translationswissenschaft [...] ausgebaut wird" (2006: 34 unter Verweis auf Schreiber 2004), und er ist damit einer von wenigen, die in gewisser Weise darauf hinweisen, dass es ganz ohne sprachwissenschaftliche Ansätze möglicherweise nicht geht. Schreiber selbst schreibt: „Für eine stärkere ,Relinguistisierung' der Übersetzungswissenschaft plädieren in jüngster Zeit vor allem Jörn Albrecht (2004) und Lew Zybatow (2004)" (Schreiber 2006: 34). Eine Relinguistisierung der Übersetzungswissenschaft, ein linguistic return, ist auch aus unserer Sicht angebracht.

Zuletzt hat nun auch House (2013) eingeräumt, dass es ganz ohne Linguistik wohl doch nicht geht; sie entwirft, nachdem sie eine Reihe von Übersetzungswissenschaftlern aufzählt, "who have maintained an interest in linguistic matters to this day", einen "new linguisticcognitive approach", , a new combination of a translation theory and a neuro-functional theory of bilingualism", dessen Notwendigkeit sie wie folgt beschreibt:

"A new linguistic-cognitive orientation in translation studies is important today because it can complement the current strong wave of socially and culturally oriented research into and around translation. For balance, it is also necessary and insightful to describe and explain how strategies of comprehending, decision-making and reverbalisation come about in a translator's bilingual mind." (House 2013: 46)

Futhark 9 (2014) Sinner,Hernández,E., Hernández,H., Ich werde ja keine, 453-466 ISSN 1886-9300 
Betrachtet man die Veröffentlichungen in der Übersetzungswis. senschaft, so erkennt man wesentliche Divergenzen, d. h. Hauptklas. sen oder -tendenzen von Texten zur Übersetzungswissenschaft. Einerseits gibt es Arbeiten, die über Übersetzungs- und Dolmetsch. wissenschaft sprechen, mit empirischer Grundlage oder ohne, und manchmal ist die empirische Grundlage dabei nicht mehr als eine Reihe von Beispielen. Andererseits gibt es Arbeiten, die über Didaktik in der Translationswissenschaft sprechen, über Qualität, Evaluierung oder Methoden der Translationswissenschaft. Man muss unterscheiden zwischen Arbeiten, die neue Erkenntnisse bringen und solchen, die Bekanntes neu darbieten, neue Namen für alte Kategorien einführen und alte Erkenntnisse neu verpackt darbieten. Sehr illustrativ sind in diesem Kontext die Beiträge von Nord (2012a und 2012b) $\mathrm{zu}$ der Frage, ob man in der Übersetzungswissenschaft mit der Suche nach "Paratranslation" gerade dabei ist, das Fahrrad wieder neu zu erfinden. Und es gibt natürlich Studien, die einfach nur bekannte Aspekte mit neuen Beispielen versehen, immer wieder neue Varianten von "das Problem der Übersetzung von $x$ am Beispiel von $y^{\prime \prime}$. Man kann schließlich noch unterscheiden zwischen Arbeiten, die übertragbare Ergebnisse bringen und Arbeiten, die das nicht tun. Und übertragbar sind naheliegenderweise vielfach nicht die Studien, die nur etwas schon Bekanntes neu illustrieren oder nur schreiben, womit man sich mal auseinandersetzen könnte, wenn es denn technisch möglich wäre.

In vielen Bereichen stoßen Übersetzungswissenschaftler ohne Linguistik an ihre kreativen Grenzen, wenn es darum geht, etwas Neues zu finden, womit sich die anderen noch nicht auseinandergesetzt haben. Und es wird aus unserer Sicht immer deutlicher, dass es vielfach linguistische Herangehensweisen sind, die neue, überaus interessante Erkenntnisse liefern.

In der Evaluierung von Übersetzungsqualität gibt es beispielsweise im Hinblick auf die Messbarkeit schon länger die Einsicht, dass man mehr tun muss als Fehler zu zählen und zu addieren bzw. eben von einer Ausgangsebene aus zu substrahieren. Natürlich gibt es mit kulturellem Wissen oder vielmehr fehlendem kulturellen Wissen erklärbare Fehler, pragmatische Fehler etc., aber es gibt eben

Futhark 9 (2014) Sinner,Hernández,E., Hernández,H., Ich werde ja keine, 453466 ISSN $1886-9300$ 
auch sprachliche Fehler, und wie man immer wieder feststellen kann, sind viele der "kulturbedingten" Fehler letztlich lediglich Lexikfehler.

Aber worum es hier vor allem geht ist die Frage der Messbarkeit. Dass man übersetzte Texte mit Originaltexten der Zielsprache vergleichen kann, ist ein Ergebnis linguistischer Studien, die mit der Korpuslinguistik immer leichter machbar sind. Stilometrische Untersuchungen sind nichts anderes als linguistische Analysen, eine Art Frequenzstudien: Häufigkeitsermittlung ist das Ergebnis linguistischer Studien. Ohne linguistische Ansätze wären viele der neuen, innovativen Arbeiten nicht möglich gewesen.

Studien zu eye tracking, der letzte Schrei in vielen Instituten, sind genau betrachtet nichts anderes als Rezeptionsanalysen, bei der die Wirkung bestimmter Strukturen geprüft wird. Es sind vielfach psycholinguistische oder quantitative linguistische Analysen, wie etwa die Studien von Kruger (2013) oder Cappelle/Loock (2013) illustrieren, in denen die Verarbeitung von verfremdeten Elementen in übersetzten Kinderbüchern analysiert wurde oder die Frequenz von existentiellem there is im Englischen und dem französischen il y $a$ in übersetzten und nicht übersetzten Texten.

Das Problem ist, aus unserer Sicht, dass die Studierenden in den Veranstaltungen zur Übersetzungstheorie oder durch die Lektüren so sie kritisch und aufmerksam lesen - lernen, dass Linguistik von gestern sei, dass lediglich Kultur und Skopos zählen, dass funktional übersetzt werden müsse. In der Praxis treffen sie dann aber auf eine bestimmte Sprachkombination, und das ist ja genau der Ort, wo es um sprachenpaarbezogene Probleme geht, wo es schön ist zu wissen, dass wir alle durch unsere Kultur geprägt sind, wo sie aber dann beim Übersetzen an konkreten Aspekten des Ausgangstextes arbeiten müssen. Natürlich gibt es dann immer Beispiele dafür, was man alles bearbeitet, was man weglässt, was man anpasst an die Zieltextkonventionen usw. Wenn man im Ausgangstext eine dreizeilige Grußfloskel hat, wo man im deutschen Mit freundlichen Grüßen schreiben würde, muss man mit den Studierenden nicht allzu lange debattieren um festzustellen, dass aus funktionalen Gründen, wegen der Textkonventionen, in der Textsorte Brief usw. im Deutschen dann eben die drei Wörter reichen. Aber all das bedeutet ja nun nicht, dass 
die Studierenden im Unterricht (und machen wir uns nichts vor: auch später im Berufsleben) nicht dennoch vor allem eins tun: einzelsprachlich bedingte Probleme beim Übersetzen lösen an den Stellen, wo sie nicht weglassen, ersetzen, kürzen, eingreifen, wo der Skopos ihnen nicht nahelegt, sich neue Dinge auszudenken, spanische Dichter durch deutsche Dichter zu ersetzen, deutsche Kinderlieder in Romananfängen durch spanische Kinderlieder zu ersetzen, weil der Skopos es so nahelegt - sondern "einfach nur übersetzen, was dasteht".

Und dann geht es nicht ohne linguistische Fähigkeiten. Ohne Sprachkenntnisse geht es ohnehin nicht, aber wenn man dann mit den Studierenden debattiert, wie man kulturbedingte Leerstellen füllt, landet man wieder bei der Linguistik. Wir glauben, dass den Studierenden falsche Signale gegeben wird, wenn nicht deutlicher gemacht wird, wie wichtig die theoretische Durchdringung ihręr Arbeitssprachen und - sprachenpaarbezogen - linguistischer Aspekte ist. Wir möchten dies an einem Beispiel illustrieren, das aus unserer Sicht paradigmatisch ist. Im Titel unseres Beitrags liest man „Ich werde ja keine Morpheme übersetzen". Es handelt sich um ein Originalzitat einer Studentin, geäußert als Reaktion auf die Auseinandersetzung mit Wortbildung. Eines der besonders gerne genannten Probleme der Übersetzung ist das der Realia; es handelt sich möglicherweise um eines der am stärksten kulturgebundenen Phänomene, aus unserer Sicht aber im wesentlichen um ein Problem, das auf der Ebene der Lexik anzusiedeln ist und mit einem der Verfahren, die von Autoren der Leipziger Schule, der Stylistique comparée, Koller usw. im Rahmen der Äquivalenzdebatten betrachtet wurden, gelöst werden kann (s. hierzu Sinner 2013; Wotjak/Sinner/Jung/Batista Rodríguez 2013).

Die Beantwortung der Frage, wie ein Übersetzer vorgehen kann, um Leerstellen zu füllen - seien es Realia, seien es neu geschaffene Konzepte aus einer Sprache, die in der anderen Sprache ausgedrückt werden müssen -, erschöpft sich im Wesentlichen mit dem, was die linguistischen Ansätze in der Übersetzungswissenschaft hierzu zu sagen hatten. Gerade der Gebrauch von lexikogenetischen Verfahren der ZS findet in der einschlägigen übersetzungswissenschaftlichen

Futhark 9 (2014) Sinner,Hernández,E., Hernández,H., Ich werde ja keine, 453-466 ISSN 1886-9300 
Literatur praktisch keine Berücksichtigung. Vor allem aber werden morphologische Kenntnisse in translatologischen Kompetenzmodellen unter den so genannten translatorischen Kompetenzen nicht gesondert aufgeführt, und sie werden auch im Zusammenhang mit den Fähigkeiten, die ein Übersetzungsstudent zur Verbesserung dieser Kompetenzen entwickeln muss, nicht explizit erwähnt. ${ }^{1}$ Keines der Modelle weist explizit auf die Notwendigkeit hin, die - „linguistische" - Kompetenz zu besitzen oder auszubilden, in der ZS noch nicht eingeführte Konzepte auszudrücken. Unerwähnt bleibt auch die damit einhergehende Verantwortung, sprachschöpferisch tätig $\mathrm{zu}$ werden, um die ZS um die expressiven Mittel zu bereichern, die für den Ausdruck der zu transferierenden Konzepte erforderlich sind. Die Lehrpläne und Studienordnungen bzw. Studienverlaufspläne der wichtigsten Ausbildungsstätten weisen - soweit wir das übersehen über Sitzungen zur Wortbildung oder Neologie in den Einführungen in die Allgemeine Sprachwissenschaft hinausgehend keine explizit der Auseinandersetzung mit der Morphologie und Neologie der ZS bzw. der Muttersprache gewidmeten Lehrveranstaltungen auf.

Die Studierenden sind nun reihenweise davon überzeugt, dass ihnen die Skopostheorie und die funktionalen Ansätze zeigen, wie sie übersetzen sollen. Auf die Frage danach, wie man nun Leerstellen damit löst, wird reihenweise mit Aussagen wie "da geht man dann so vor, dass die Loyalität nicht verletzt wird" oder , ,je nach Auftrag", , ,je nach Skopos" usw. geantwortet. Obwohl die Studierenden im prak-

\footnotetext{
${ }^{1}$ Die Frage nach der translatologischen Kompetenz ist vor allem in Publikationen behandelt worden, die sich mit der Didaktik der Übersetzung und des Dolmetschens auseinandersetzen, und es gibt eine lange Reihe von Publikationen, die sich auf die eine oder andere Weise an die Kompetenz annähem. In fast allen dieser Arbeiten wird neben translatologischen Kompetenzen (oder, je nach Fall, Teilkompetenzen oder Unterkompetenzen) wie der kommunikativ-pragmatischen, der stilistischen, registerbezogenen, textuellen, kognitiven und kulturellen Kompetenz die sprachliche bzw. linguistische Kompetenz ohne eine über eine Unterteilung in Kompetenz in der AS und Kompetenz in der ZS hinausgehende Differenzierung aufgeführt. Für das Beispiel der Leipziger Schule könnten hier z. B. syntaktisch-semantische und kommunikativ-pragmatische Subkompetenz genannt werden, für die PACTE-Gruppe zweisprachige Subkompetenz, die wiederum untergliedert wird in pragmatische, soziolinguistische, textlinguistische und lexikogrammatische Kenntnisse (s. PACTE 2007).
} 
tischen Ubersetzungsunterricht lexikogenetische Verfahren zur Schaf. fung von Ausdrücken bzw. neuen Termini kennengelernt haben, wird die übersetzungswissenschaftliche Tendenz der Zurückweisung der linguistischen Ansätze fast mechanisch ubernommen und erst überdacht, wenn man sie darauf hinweist, dass sie gerade auf Grundlage linguistischer Verfahren ein Problem gelöst haben, für das die funktionalen Ansätze nur eine Kategorie, aber keinen Lösung. sansatz anbieten.

In den mündlichen Prüfungen in Übersetzungswissenschaft fragten wir - Elia Hernández und Carsten Sinner etwa in der Modulabschlussprüfung Translationswissenschaft im Sommer 2013 - die Studierenden danach, welche Theorien ihnen besonders praxisrelevant erscheinen. Trotz der am Institut für Angewandte Linguistik und Translatologie (IALT) immer ausführlich betrachteten linguistischen Aspekte ist die häufigste Antwort, dass für die Praxis die Skopostheorie und das funktionale Übersetzen am relevantesten seien, da sie dem Übersetzer sagen, wie er vorzugehen habe. Tatsächlich gilt das für die Vorbereitung einer Übersetzung: die Bestimmung des Zwecks einer Übersetzung, die Frage, wie es um den Loyalitätsanspruch bestellt sein könnte, die anzuwendende Übersetzungsmethode, die Notwendigkeit der Bearbeitung inhaltlicher Art usw. Aber auf der Ebene der einzelsprachlichen bzw. der sprachpaarbezogenen Probleme sind diese Aspekte natürlich kaum als Lösungsansatz zu verstehen. Auf die Frage nach den Ansätzen zur Lösung von sprachpaarbezogenen Problemen - die auch die Funktionalistin Nord in ihrer Klassifizierung von Übersetzungsproblemen und Übersetzungsschwierigkeiten erwähnt (s. Nord 1987) - und danach, wie genau die funktionale oder die skoposorientierte Übersetzungswissenschaft hier Lösungen anbiete bzw. welches genau die Instruktionen dieser Ausrichtungen sind, oder danach, wie man mit diesen Problemen umgehen könnte, sind die Studierenden immer wieder überfragt. Betrachtet man die Darstellung in den gängigen Einführungen, sieht man schnell, dass sie lediglich eine Grundhaltung der modernen Übersetzungswissenschaft reproduzieren, die aus Gründen ein Forschungs- und Lehrparadigma ausgrenzen, die uns vor allem ideologisch erscheinen.

Futhark 9 (2014) Sinner,Hernández,E., Hernández,H., Ich werde ja keine, 453-466 ISSN $1886-9300$ 
guistischen Herangehensweisen an die Übersetzung in Einführungen dargestellt wird, die Studierenden zu der Schlussfolgerung bringt, dass Linguistik in der Ubersetzung keine Relevanz hat, "überwunden" kann. Entsprechend negativ ist der Blick Lösungen "anbieten auf sprachwissenschaftliche Lehre Blick der Studierenden auf in der Einführung in Studierenden in der Einführung in die allgemeine Sprachwissenschaft, wo unter vielen anderen Aspekten die Wortbildung betrachtet und auch zwischen der Wortbildung in den Arbeitssprachen der studierenden und im Deutschen verglichen wird, sind gemäß der Befragungen zum Ende des Semesters in den letzten fünf Jahren der Meinung gewesen, dass die Lehrveranstaltung keinen wesentlichen Bezug zur Übersetzung aufweist. In den Begründungen in den Freifeldern wurde $u$. a. darauf hingewiesen, dass der linguistische Ansatz in der Übersetzungswissenschaft längst als überholt angesehen werde und statt einer Einführung in die Linguistik besser mehr kulturwissenschaftliche Veranstaltungen angeboten werden sollten.

Neben einem allgemeinen Problem der Ausbildung von DolmetscherInnen und ÜbersetzerInnen, den vielfach sehr stark auszubauenden Sprachfertigkeiten, spielt unserer Meinung nach die fehlende Insistenz der Übersetzungswissenschaft auf der Notwendigkeit von Kenntnissen bzw. Grundlagen in der Sprachwissenschaft hier eine wichtige Rolle. Dies betrifft manche Ausbildungszentren mehr als andere. Am IALT in Leipzig sind wir in der privilegierten Lage, dass unsere Studierenden sehr gute Sprachkenntnisse der Fremdsprachen besitzen müssen, wenn sie ihr Studium aufnehmen, und auch eine Deutschprüfung bestehen müssen, also auch gute Kenntnisse der Muttersprache nachweisen müssen. Dies erleichtert es, aus einer sprachwissenschaftlichen Perspektive übersetzungsrelevante Aspekte zu betrachten. Es ist uns bewusst, dass dies für Institute (wie etwa sehr viele Einrichtungen in Spanien), an denen die Studienanfänger keine oder wenige Fremdsprachkenntnisse mitbringen, bedeutet, dass es noch schwieriger ist, eine linguistische Sicht auf Übersetzung zu berücksichtigen. 
Im Interesse der Studierenden und - langfristig gesehen - im Interesse der Übersetzungsqualität und der Forschung muss es jedoch eine linguistische Kehrtwende geben. Olnne linguistische Analysen und Betrachtungen, alleine mit Debatten um Kultur, Interkulturalität, Skopos und die Situationsgebundenheit des handelnden Übersetzers als Experte wird man den Herausforderungen der Übersetzung und den stetig wachsenden Anforderungen der Übersetzungsmärkte nur in engen Grenzen gerecht werden können.

Wesentliche Fragen der Qualität der Übersetzung und der Übersetzungsevaluation (und das sind dringende und angesichts der zunehmenden Präsenz und Zuverlàssigkeit maschineller bzw. automatisierter Übersetzungsverfahren auch "überlebenswichtige“ Fragen) sind nur durch Vergleiche der Eigenheiten von Originaltexten der Zielsprache und übersetzten Texten und durch vergleichende Rezeptionsstudien zu beantworten. Wer das anders als mit linguistischen Verfahren versuchen will, wird über Allgemeinplätze und Vermutungen und das bloße Illustrieren von Aussagen mit Beispielen, die weder repräsentativen noch signifikanten Studien entspringen, nicht weit kommen.

Es geht uns nicht darum, die Bedeutung von Kulturgebundenheit und Skopos in Frage zu stellen, nur denken wir, dass die Zukunft in der Übersetzungswissenschaft in einer Rückkehr zu "exakteren" Techniken zu suchen ist, die eine Messung erlauben; sie sind durch die kulturelle und pragmatische Wende etwas aus den Augen geraten und wären nun in die Didaktik und Forschung wieder befruchtend einzubringen. Die Möglichkeiten der Korpuslinguistik und Stilometrie zeigen eindrucksvoll, welche Chancen sich durch die sprachwissenschaftliche Analyse in der Translatologie bieten. Es wäre leichtsinnig, aus ideologischen Gründen auf die Linguistik als Grundlage und Handwerkszeug zu verzichten.

Sie wissen, dass wir am IALT noch immer viel mit Linguistik arbeiten; wie der Name ja sagt, sind die Aufgabenbereiche des IALT Angewandte Linguistik und Translatologie. Es geht uns hier nicht um Theorien "auf dem Papier", sondern um eine eigentlich generalisierbare Philosophie an unserem Institut; diese wird in unserer Arbeit durch die starken Interrelationen zwischen Terminologie und Transla-

Futhark 9 (2014) Sinner,Hernández,E., Hernández,H., Ich werde ja keine, 453-466 ISSN $1886-9300$ 
tion, Textlinguistik und Úbersetzung, Grammatik und Translation, varietätenlinguistischen Fragestellungen und Translatologie usw. sichtbar.

Wir glauben, dass man die Gewinne der kulturellen und pragmatischen Wende nur umsetzen bzw. nutzen kann, wenn die Errungenschaften der ihr vorausgehenden linguistischen Ansätze nicht aufs Spiel gesetzt werden: Die kulturelle Wende war nur möglich, weil die linguistischen Herangehensweisen die Grundlagen gelegt hatten. Umgekehrt sind aber ohne diese linguistischen Grundlagen die Früchte der ihnen folgenden Ansätze nicht mehr erreichbar.

\section{BIBLIOGRAPHISCHE ANGABEN}

ALBRECHT, Jörn, „Der Beitrag der Übersetzungswissenschaft zur Übersetzungsforschung", in: ALBRECHT, Jörn; GERZYMISCH-ARBOGAST Heidrun; ROTHFUB-BASTIAN, Dorothee (Hrsg.), Übersetzung - Translation Traduction. Neue Forschungsfragen in der Diskussion. Festschrift für Werter Koller, Tübingen, Narr, 2004, 1-21.

ALBRECHT, Jörn, Grundlagen der Übersetzungsforschung. Übersetzung und Linguistik, Tübingen, Narr, 2005.

CAPPELLE, Bert; LOOCK, Rudy, „Is there interference of usage constraints?: A frequency study of existential there is and its French equivalent il $y a$ in translated vs. non-translated texts", Target 25, 2, 2013, 252-275.

HOUSE, Juliane, „Towards a new linguistic-cognitive orientation in translation studies", Target 25, 1, 2013, 46-60.

KOLLER, Werner, Einfïlhrung in die Übersetzungswissenschaft, 6., durchgesehene und aktualisierte Auflage, Wiebelsheim, Quelle und Meyer, 2011.

KRUGER, Haidee, "Child and adult readers' processing of foreignised elements in translated South African picturebooks: An eye-tracking study", Target $25,2,2013,180-227$.

NORD, Christiane, "Translation und Paratranslation. Wie man das Fahrrad immer wieder mal neu erfinden kann", Lebende Sprachen 57, 2012a, 1,1-8.

NORD, Christiane, „Paratranslation - a new paradigm or a re-invented wheel?", Perspectives: Studies in Translatology 20, 4, 2012b, 399-409.

NORD, Christiane, „Übersetzungsprobleme - Übersetzungsschwierigkeiten. Was in den Köpfen voN Übersetzern vorgehen sollte...", Mitteilungsblatt für Dolmetscher und Übersetzer, 1987, 2, 5-8.

PACTE, „Zum Wesen der Übersetzungskompetenz", in: Gerd Wotjak (Hrsg.) (2007): Quo vadis Translatologie? Ein halbes Jahrhundert universitärer Ausbild-

Futhark 9 (2014) Sinner,Hernández,E., Hemández,H., Ich werde ja keine, 453-466 ISSN $1886-9300$ 
ung von Dolmetschern und Übersetzern in Leipzig. Rückschan, Zwischenbilanz und Perspektiven aus der Außensicht, Berlin, Frank \& Timme, 2007, págs. . $327-342$.

SCHREIBER, Michael, „Kontrastive Linguistik und sprachpaarbezogene Trans. lationswissenschaft", in: GYDE Hansen; MALMKJÆR, Kirsten; GILE, Daniel (Hrsg.), Claims, Changes and Challenges in Translation Studies, Amsterdam/Philadelphia, Benjamins, 2004, 83-98.

SCHREIBER, Michael, Grundlagen der Ubersetzungswissenschaft. Franzosisch, Italienisch, Spanisch, Tübingen, Niemeyer, 2006.

SINNER, Carsten, „Indem ich übersetze, übernehme ich Verantwortung. Über die Verantwortung des Übersetzers für seine Sprache", in: ENDE, AnneKathrin; HEROLD, Susann; WEILANDT, Annette (Hrsg.), Alles hängt mit allem zusammen. Translatologische Interdependenzen. Festschrift für Peter A. Schmitt, Berlin, Frank \& Timme, 2013, 251-282.

SINNER, Carsten, „El problema de la recreación de los compuestos alemanes en las lenguas iberorrománicas: a proposito del término Laienlinguistik en la transmisión intercultural del saber", in: TABARES PLASENCIA, Encarnacion; IVANOVA, Vessela; KRÜGER, Elke (Hrsg.), Análisis lingüístico contrastivo de textos especializados en español y alemán, Berlin, Frank \& Timme, 2008, 155181.

SNELL-HORNBY, Mary (Hrsg.), Übersetzungswissenschaft. Eine Neuorientierung. Zur Integration von Theorie und Praxis, 2. Auflage. Tübingen, Francke, 21994 [1986].

Truffaut, Louis, Problèmes linguistiques de traduction, München, Hueber, 1983. ZYBatOW, Lew, "Quo vadis, Translationswissenschaft?", in: FLeISCHMANN, Eberhard; SCHMITT, Peter A.; WOTJAK, Gerd (Hrsg.), Translationskompetenz, Tübingen, Stauffenburg, 2004, 287-308.

Futhark 9 (2014) Sinner,Hernández,E., Hernández,H., Ich werde ja keine, 453-466 ISSN 1886-9300 